ARTICLE

Received 2 Oct 2013 | Accepted 5 Feb 2014 | Published 4 Mar $2014 \quad$ DOl: 10.1038/ncomms4384

\title{
Environment-responsive nanophores for therapy and treatment monitoring via molecular MRI quenching
}

Charalambos Kaittanis ${ }^{1}$, Travis M. Shaffer ${ }^{1,2}$, Anuja Ogirala ${ }^{1}$, Santimukul Santra ${ }^{3}$, J. Manuel Perez ${ }^{4}$, Gabriela Chiosis ${ }^{1}$, Yueming $\mathrm{Li}^{1}$, Lee Josephson ${ }^{5}$ \& Jan Grimm ${ }^{1}$

The effective delivery of therapeutics to disease sites significantly contributes to drug efficacy, toxicity and clearance. Here we demonstrate that clinically approved iron oxide nanoparticles (Ferumoxytol) can be utilized to carry one or multiple drugs. These so called 'nanophores' retain their cargo within their polymeric coating through weak electrostatic interactions and release it in slightly acidic conditions ( $\mathrm{pH} 6.8$ and below). The loading of drugs increases the nanophores' transverse T2 and longitudinal T1 nuclear magnetic resonance (NMR) proton relaxation times, which is proportional to amount of carried cargo. Chemotherapy with translational nanophores is more effective than the free drug in vitro and in vivo, without subjecting the drugs or the carrier nanoparticle to any chemical modification. Evaluation of cargo incorporation and payload levels in vitro and in vivo can be assessed via benchtop magnetic relaxometers, common NMR instruments or magnetic resonance imaging scanners.

\footnotetext{
${ }^{1}$ Molecular Pharmacology and Chemistry Program, Memorial Sloan Kettering Cancer Center, 1275 York Avenue, New York, New York 10065, USA.

${ }^{2}$ Department of Chemistry, Hunter College of the City University of New York, Graduate Center, New York, New York 10065, USA. ${ }^{3}$ Department of Chemistry, Pittsburg State University, 1701 S Broadway Street, Pittsburg, Kansas 66762, USA. ${ }^{4}$ NanoScience Technology Center, University of Central Florida, 12424 Research Parkway, Suite 400, Orlando, Florida 32826, USA. ${ }^{5}$ Center for Advanced Medical Imaging Sciences, Massachusetts General Hospital, Building 149, 13th Street, Charlestown, Massachusetts 02129, USA. Correspondence and requests for materials should be addressed to J.G. (email: grimmj@mskcc.org).
} 
T he effective delivery of therapeutics is critical for treatment. Drugs must stay in circulation for adequate time, avoiding clearance by the liver and kidneys and achieve sufficiently high accumulation in the site of the disease, in order to maximize therapeutic efficacy and minimize side effects. Towards this goal, researchers have utilized innovative strategies, including the modification of drugs like Doxorubicin with polymers and targeting moieties, in order to achieve delivery to tumours via the enhanced permeability and retention effect or the targeting of overexpressed surface markers ${ }^{1-4}$. Apart from receptors involved in signal transduction, nutrient receptors, such as the folate or transferrin receptor, were targeted for the delivery of chemotherapies ${ }^{5-7}$. Furthermore, in order to determine the distribution of these molecular constructs, tracers or fluorochromes were covalently conjugated to them ${ }^{8-11}$. However, since the parent drugs have undergone modifications, including addition of new bonds, functional groups and entire molecules to either achieve targeting or monitoring, these therapeutic agents face extensive scrutiny from regulatory bodies $^{12-14}$

An appealing alternative is the encapsulation of therapeutics within the nanoparticles that provide aqueous stability and longer circulation times, without subjecting the drug to any chemical modification ${ }^{15-17}$. For instance, liposomal formulations of chemotherapeutics and antifungals, like Doxorubicin (Doxil) and Amphotericin B (AmBisome), are in clinical use and provide improved pharmacokinetics and ability to deliver high loads of drugs with otherwise poor aqueous solubility. Drug delivery occurs upon fusion with the plasma membrane or action of lipases ${ }^{18,19}$. Alternatives to liposomes are nanoparticles that consist of polymers, like poly(lactic-co-glycolic) acid and hyperbranched polyesters, which can be hydrolysed in vivo by enzymes, like esterases, and acidic conditions $\mathrm{s}^{20-22}$. These nanoparticles delivered drugs, like Taxotere, in cultured cells and animal models. The encapsulation process resulted in loading of the drugs within the nanoparticle's cavity and allowed the use of the nanoparticle's surface functional groups for further bioconjugation of targeting moieties. However, although targeted nanoparticles can be developed for both drug delivery and imaging via clinical diagnostic modalities, these nanoparticles have not been investigated by regulatory agencies ${ }^{23}$.

Iron oxide nanoparticles (IONP) formulations have been used as contrast agents for magnetic resonance imaging (MRI). Currently, Ferumoxytol (Feraheme) is used in the clinic for the treatment of iron deficiency. These nanoparticles consist of iron and a carbohydrate (dextran) and are well tolerated, without any side effects and toxicity. Therefore, we investigated whether Ferumoxytol can serve as a magnetic drug carrier suitable to carry several hydrophobic drugs after facile loading through coincubation and improve their therapeutic efficacy, without further modification of either the nanoparticle or drugs. Such a drug delivery system promises to move faster to the clinic, since it is based on an already clinically approved vehicle (Ferumoxytol) and simply takes up the drug without chemical reactions; therefore, the drug-loaded particles were aptly termed nanophores. In addition, developing a facile method to load different drugs on a common delivery platform has the unique potential of being readily adopted in clinic. We also hypothesized that the drug loading could be monitored through MRI, since IONP's magnetic properties have been previously used in sensitive assays. These assays rely on the nanoparticles' ability to affect the proton nuclear magnetic resonance (NMR) signal of the surrounding water molecules. Specifically, IONPs primarily affect the transverse (spin-spin; T2) relaxation time of bulk water protons, facilitating sensitive quantification and imaging with compact relaxometers, NMR or MRI instruments ${ }^{24-30}$.
Herein, we report that magnetic nanophores can be selfreporting delivery vehicles for many chemotherapeutics. When the cargo is loaded, the nanoparticle's magnetic properties are affected and the relaxation times T2 and T1 increase. Diffusion MRI, which allows the measurement of water diffusion in a given voxel through the apparent diffusion coefficient, revealed that the cargo hinders water's diffusion within the nanoparticles' coating as probable reason for the induced changes in relaxivity. After establishing this optical-independent method to characterize drug-loaded nanophores, we show that drug-loaded Ferumoxytol delivers a more efficient therapy than free drugs in vitro and most importantly in vivo, for the rational inhibition of select oncogenic pathways.

\section{Results}

Clinical IONP as drug nanophores. Considering the need for translational drug delivery platforms and the already existing clinical use of Ferumoxytol, we first investigated whether Ferumoxytol could be used as a nanophore for the delivery of drugs. Hence, we encapsulated therapeutics and fluorophores within Ferumoxytol, using the solvent diffusion method, in order to facilitate the facile entrapment of hydrophobic molecules within Ferumoxytol's carboxymethyl dextran coating. After dialyzing the nanoparticles in order to remove any free cargo from the solution, we determined that Ferumoxytol could carry different amounts of the fluorescent Taxol analogue Flutax1 (MW: 1,337), without affecting the nanoparticles' physical properties (Fig. 1a; Supplementary Fig. 1a,b). The encapsulation process had a minimum of $80 \%$ loading efficiency for Flutaxl, as determined through ultraviolet-vis spectroscopy after dialysis of the Flutax1loaded nanophores in order to remove free cargo, suggesting that large amounts of payload could be entrapped within Ferumoxytol's coating (Fig. 1b). Furthermore, we demonstrated that Ferumoxytol could also carry smaller compounds, such as the near-infrared fluorophore DiR and the chemotherapeutic Doxorubicin, with molecular weights of 1,013 and 580, respectively. Dynamic light scattering (DLS) analysis revealed that drug loading did not affect the average diameter of Ferumoxytol, indicating that the cargo and the encapsulation process do not affect the nanoparticles' size (Fig. 1c). Apart from the clinical Ferumoxytol, these compounds were effectively loaded in inhouse synthesized nanoparticles, such as poly(acrylic acid) (PAA)-coated and aminated IONP, with the mean diameter of the loaded nanoparticles being similar to that of the unloaded (vehicle) nanoparticles (Supplementary Fig. 1c,d), indicating that the loading capability is not unique to Ferumoxytol. Since serum stability is a key parameter in drug delivery, we utilized DiR-loaded Ferumoxytol and determined that the nanoparticles' near-infrared fluorescence did not significantly change after prolonged incubation in serum (Fig. 1d), with the formulation being uniformly suspended and lacking any signs of aggregation (Supplementary Fig. 1e).

The serum stability of cargo-loaded Ferumoxytol prompted us to study whether the cargo could be retained at physiological conditions but released upon sensing the reduced $\mathrm{pH}$ in many tumours. This feature is ideal for cancers that exhibit decreased interstitial $\mathrm{pH}$, due to upregulated glycolysis as a result of signalling and metabolic alterations ${ }^{31}$. In order to examine the use of Ferumoxytol as a microenvironment-responsive drug delivery system, we studied the retention of Doxorubicin by Ferumoxytol at physiological and lower $\mathrm{pH}$ levels as seen in tumours. We employed a dialysis chamber to separate the nanoparticles from the potentially released drug. Since Doxorubicin is fluorescent ${ }^{11}$, we monitored the fluorescence emission of Doxorubicin-loaded Ferumoxytol, as well as the 

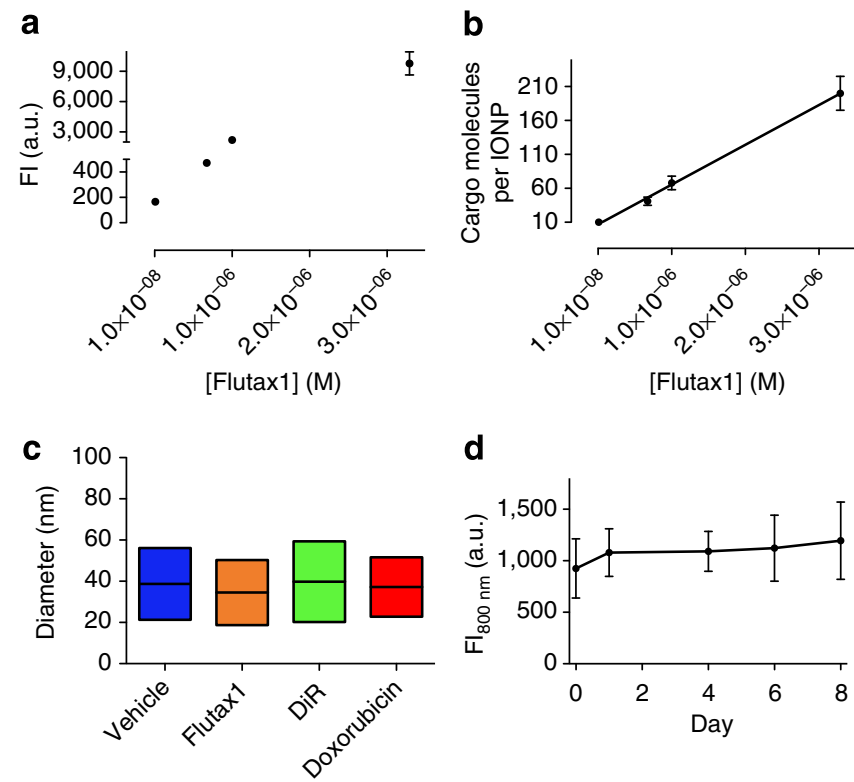

d
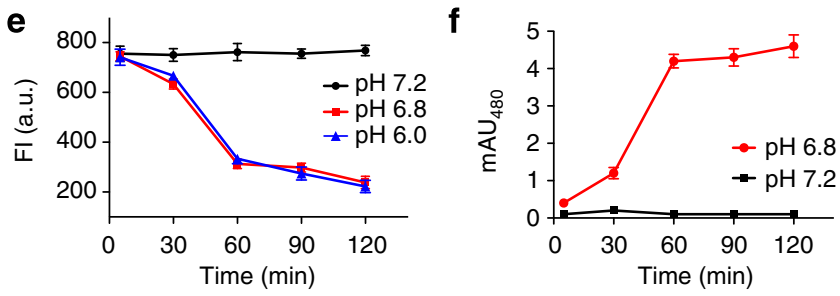

Figure 1 | Ferumoxytol as nanophores for drug delivery. (a) The gradual incorporation of Flutax1 within Ferumoxytol's coating increased the nanoparticles' Flutax1-derived fluorescence emission (mean \pm s.e.m., $n=3$ ), (b) with the nanoparticle coating capable of accommodating many cargo molecules within it (mean \pm s.e.m., $n=3$ ). The nanoparticles were first dialysed to remove any unloaded compound, followed by fluorescence and DLS measurements. (c) Size distribution of cargo-loaded Ferumoxytol (vehicle = unloaded Ferumoxytol; means and distributions of three independent experiments). (d) DiR-loaded Ferumoxytol was stable in sterile fetal bovine serum, with its fluorescence remaining unaltered (mean \pm s.e.m., $n=3$ ). (e) Ferumoxytol released Doxorubicin at slightly acidic conditions. The fluorescence emission of the nanoparticles

( $\lambda_{\mathrm{ex}}=485 \mathrm{~nm}, \lambda_{\mathrm{em}}=590 \mathrm{~nm}$, mean \pm s.e.m., $n=3$ ) that were retained within the dialysis chamber decreased, due to Doxorubicin's release to the free fraction found in the chambers' exterior. (f) The release of Doxorubicin from Ferumoxytol to the exterior of the dialysis chamber was confirmed by recording the drug's absorbance in the free fraction at $480 \mathrm{~nm}$ (mean \pm s.e.m., $n=3$ ).

presence of free drug outside the dialysis chamber via HPLC-based spectrophotometry. We determined that while Ferumoxytol retained Doxorubicin at $\mathrm{pH} 7.2$, it released the drug at slightly lower $\mathrm{pH}$ (Fig. 1e,f).

We next hypothesized that the release of cargo at lower $\mathrm{pH}$ was facilitated due to disruption of the weak electrostatic interactions that mediate the association of the drug with the nanoparticles' polymeric coating by the increased positive protons. Changes in the protonation of the polymer's side chains disturb hydrogen bonding and van der Waals interaction between Ferumoxytol's coating and the cargo, thus triggering release at lower $\mathrm{pH}$. Although Doxorubicin can form amine salts that improve its water solubility, this occurs at significantly lower $\mathrm{pH}$, with concomitant changes in the solution's colour (from orange-red at neutral $\mathrm{pH}$ to yellow-orange at acidic $\mathrm{pH}$ ) that were not observed at the $\mathrm{pH}$ levels of our study, as the solutions retained their orange-red appearance. To confirm that the loading of the cargo into Ferumoxytol is due to weak electrostatic interactions, we attempted to load the nanoparticles with Doxorubicin in solutions with varying ionic strength. Fluorescence spectroscopy studies showed that at salt concentrations of $2 \mathrm{M}$ and above, almost no Doxorubicin was loaded into the nanoparticles (Supplementary Fig. 2a), with little changes in the nanoparticle size (Supplementary Fig. 2b). We also demonstrated that the cargo retention process is reversible, as demonstrated in $\mathrm{pH}$ based release studies, where after unloading of Doxorubicin at $\mathrm{pH} 6.8$, the same nanoparticle preparation was re-loaded with the fluorophore DiR at pH 7.4 (Supplementary Fig. 2c).

The cargo affects the nanophores' magnetic properties. While the utilized agents so far were fluorescent, this is not true for the majority of pharmaceuticals. We reasoned that having a facile non-optical method to characterize nanophore loading might have important pharmaceutical implications, especially when the drug is not fluorescent. Furthermore, such a method could provide an option to noninvasively monitor drug delivery in vivo. We evaluated therefore, if the loading of cargo into IONP-based nanophores would change their relaxivity due to displacement of water molecules from the vicinity of the IONP's iron oxide core. We utilized a benchtop relaxometer and loaded Ferumoxytol with the fluorescent Taxol derivative $\left([\text { Flutax } 1]_{\text {Ferumoxytol }}=30 \mu \mathrm{M}\right)$, Doxorubicin $\quad\left([\text { Doxorubicin }]_{\text {Ferumoxytol }}=828 \mu \mathrm{M}\right)$ and $\mathrm{DiR}$ $\left([\mathrm{DiR}]_{\text {Ferumoxytol }}=920 \mu \mathrm{M}\right)$, observing cargo-modulated alterations in the T2 and T1 signal (Fig. 2a,b). Interestingly, we observed that as the drug content in the nanophores increased (with the particle concentration being constant), the T1 and T2 relaxation times rose also over those of the unloaded nanoparticles $(\mathrm{T} 1=402 \pm 7 \mathrm{~ms} ; \mathrm{T} 2=121 \pm 2 \mathrm{~ms}$; mean \pm s.e.m; $n=3)$ (Fig. 2c). This demonstrates the use of this method for the quantification of non-fluorescent compounds loaded in IONP with simple relaxometers, NMR instruments or MR imagers (Supplementary Fig. 3a,b). Since T2 and T1 are inversely proportional to a contrast agent's spin-spin $\left(r_{2}\right)$ and spin-lattice $\left(r_{1}\right)$ relaxivities, Ferumoxytol's $r_{2}$ and $r_{1}$ decreased after addition of the cargo (Fig. 2d,e). To demonstrate that the loading of nonfluorescent drugs can be monitored through the changes in IONP's magnetic properties, we encapsulated a variety of cancer chemotherapeutics and observed similar changes in $r_{2}$ and $r_{1}$ (Fig. 2d,e; Supplementary Table 1; $[\text { Drug }]_{\text {Ferumoxytol }}=100 \mu \mathrm{M}$ ). We further determined that the changes in the magnetic properties depended on the drug's dimethylsulphoxide (DMSO) solubility (Fig. 2e), where compounds that were highly soluble in DMSO caused large changes in Ferumoxytol's relaxivity, likely due to enhanced retention by the nanoparticles' coating. This showed that the hydrophobicity of the drug was an important factor next to its size for its capability to be loaded into the nanophores. Relaxivity changes were also observable when Ferumoxytol was simultaneously loaded with two drugs at the same time, such as the androgen receptor antagonist MDV3100 and the PI3K inhibitor BEZ235 ([MDV3100 $]_{\text {Ferumoxytol }}=250 \mu \mathrm{M}$ and $\left.[\mathrm{BEZ235}]_{\text {Ferumoxytol }}=75 \mu \mathrm{M}\right) \quad$ (Fig. 2d,e). These drugs were chosen together rationally since the androgen receptor pathway interacts with the PI3K cascade in prostate cancer, suggesting that combinatorial approaches targeting both pathways will result in a more effective therapy ${ }^{32}$. Notably, although the extent of cargo loading had an effect on T2 and T1, it did not affect T2*, which was solely dependent on the iron and therefore particle concentration. Loading and unloading of Ferumoxytol can therefore be evaluated via the T2 and T1 parameters, while the required information on the relative nanophores concentration (to exclude that changes in $\mathrm{T} 2$ or $\mathrm{T} 1$ are barely due to different 
a
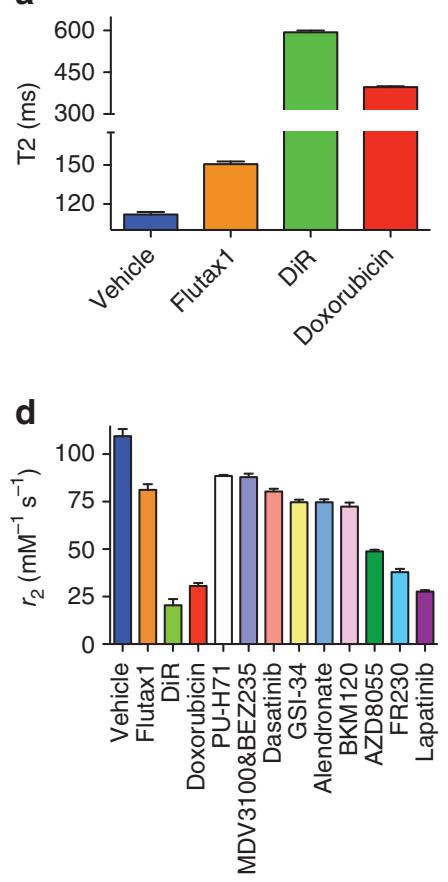

b

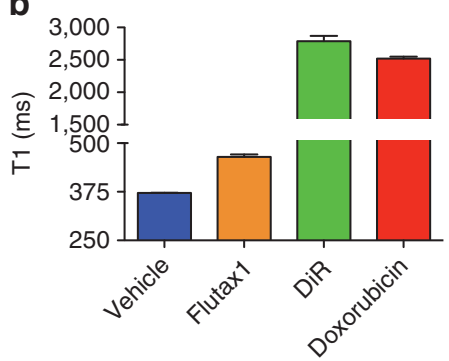

e

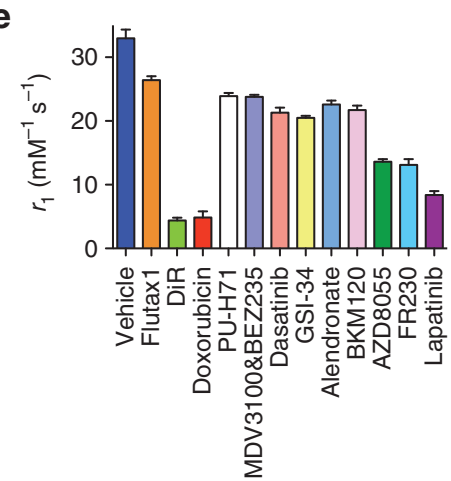

C

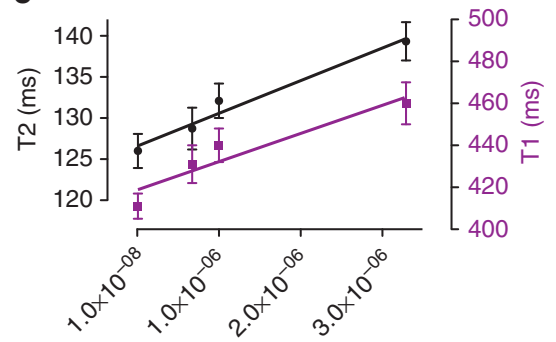

[Flutax1] (M)

$\mathbf{f}$

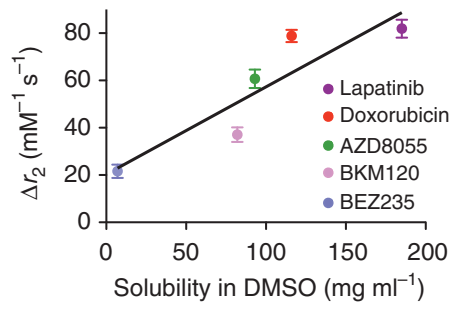

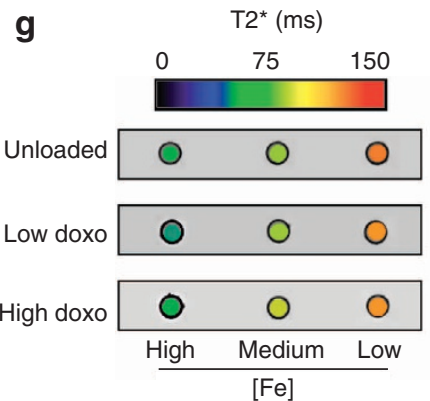

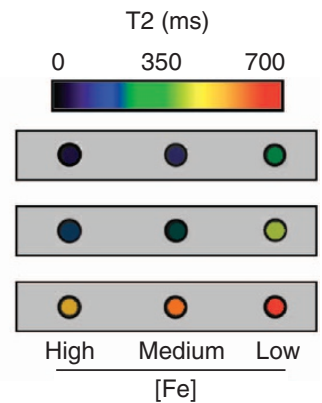

Figure 2 | The cargo quenches the nanophores' magnetic properties. Loading nanophores with cargo increased the T2 (a) and T1 (b) $([\mathrm{Fe}])=10 \mu \mathrm{g} \mathrm{ml}^{-1}$; mean \pm s.e.m., $n=3$ ). The nanoparticles were first dialyzed to remove any unloaded compound, followed by relaxation measurements. (c) The gradual addition of Flutax 1 within Ferumoxytol's coating increased the nanoparticle formulation's T2 and T1 signal (linear regression correlation coefficients $r_{\mathrm{T} 2}=0.98$ and $r_{\mathrm{T} 1}=0.94 ;[\mathrm{Fe}]=10 \mu \mathrm{g} \mathrm{ml}{ }^{-1}$; mean \pm s.e.m., $n=3$ ). (d,e) The incorporation of cargo within the nanophores' coating resulted in changes on the nanoparticles' relaxivities (mean \pm s.e.m., $n=3$ ). (f) The change in relaxivity $\left(\Delta r_{2}\right)$ was associated with the drug's solubility in DMSO (linear regression correlation coefficient $r=0.90$; mean \pm s.e.m., $n=3$; solubility information was obtained from Selleck Chemicals). (g) MRI phantom images of unloaded and loaded Ferumoxytol, demonstrating that the cargo does not affect the nanoparticles' $\mathrm{T}^{\star}{ }^{\star}$ signal, as opposed to its effect on T2 (iron concentrations: high $=10 \mu \mathrm{gll}^{-1}$, medium $=6 \mu \mathrm{g} \mathrm{ml}^{-1}$ and low $=4 \mu \mathrm{g} \mathrm{ml}^{-1}$ ).

nanophore concentration) can be provided through the $\mathrm{T}^{*}$ signal (Fig. 2g; Supplementary Fig. 4a-d). In addition, we tested drug loading in non-clinical IONP preparations, such as PAA-coated nanoparticles, and observed that the cargo affected their magnetic properties similar to what was seen in Ferumoxytol, indicating that the effect of cargo on the nanoparticles' magnetic properties is shared among polymercoated IONP (Supplementary Fig. 5a-f).

Using MR to monitor cargo release from the nanophores. Similar to the prior optical measurements, incubation of Doxorubicin-loaded Ferumoxytol at physiological conditions for $24 \mathrm{~h}$ did not reveal any major changes in the T2, T1 to indicate loss of cargo over time (Supplementary Fig. 6a-c). However, changes in T2 and T1 were observed at the slightly acidic $\mathrm{pH}$ of 6.8. Again employing a dialysis chamber to separate the nanoparticles from any released drug, we incubated Doxorubicincarrying Ferumoxytol in $1 \times$ phosphate-buffered saline (PBS) adjusted to $\mathrm{pH} 6.8$ and 6.0. Rapid decreases in T2 and T1 were again observed in these mildly acidic conditions (Fig. 3a,b), which were in accordance with the loss of nanoparticle-associated Doxorubicin fluorescence due to release of the drug to the dialysis-free fraction (Fig. 1e,f). In control studies, unloaded Ferumoxytol at pH 6.8 and below did not exhibit any changes in T2 and T1 (Fig. 3c,d), indicating that the observed changes in relaxation times of the loaded Ferumoxytol were attributed to cargo release and not due to the $\mathrm{pH}$ directly affecting the nanoparticles' magnetic properties. To confirm that these changes were mediated by cargo release and not aggregation of particles, we performed DLS-based size measurements. Results indicated that nanoparticle size and distribution were constant throughout the experiment, with the nanoparticles being stable after $2 \mathrm{~h}$ at $\mathrm{pH}$ 6.0 and both Doxorubicin-loaded and unloaded Ferumoxytol showing the same size distribution profiles (Fig. 3e,f). This demonstrates that the effect of cargo encapsulation on IONP is reversible, since payload release facilitates recovery of their magnetic properties, leading to T2 and T1 decreases. 
a

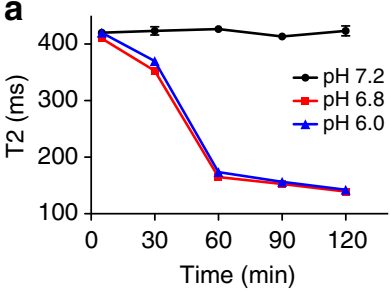

C

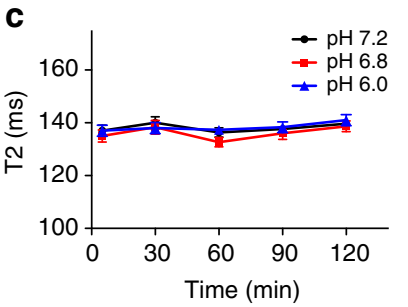

e Doxorubicin-loaded feraheme

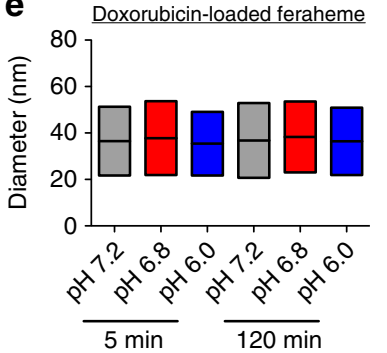

b

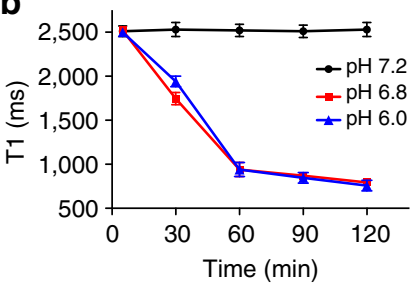

d

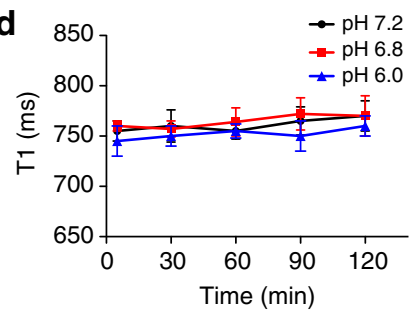

f

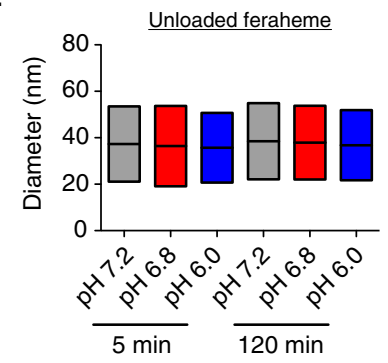

Figure 3 | The nanophores release their cargo at mildly acidic conditions. As Ferumoxytol released Doxorubicin in acidified buffers, the T2 (a) and $\mathrm{T} 1$ decreased (b) ( $[\mathrm{Fe}]=10 \mu \mathrm{g} \mathrm{ml}^{-1}$; mean \pm s.e.m., $n=3$ ). The T2 (c) and $\mathrm{T} 1$ (d) of Ferumoxytol (unloaded nanoparticles) were not affected by the slightly acidic $\mathrm{pH}\left([\mathrm{Fe}]=10 \mu \mathrm{g} \mathrm{ml}^{-1}\right.$; mean \pm s.e.m., $n=3$ ). (e) No changes in the nanoparticle size were observed via DLS during cargo release, suggesting structural integrity of Ferumoxytol in these conditions (means and distributions of three independent experiments). (f) Stability of unloaded Ferumoxytol at different $\mathrm{pH}$ (means and distributions of three independent experiments). (Middle horizontal line of a rectangle $=$ the sample's mean diameter; upper and lower horizontal lines are the boundaries of the nanoparticles' Gaussian distribution.)

Next, we investigated the long-term stability of drug-loaded nanophores in serum. DiR-carrying Ferumoxytol was stable for up to 8 days in sterile serum (Supplementary Fig. 7a,b), with no changes in the relaxation times. However, Doxorubicin- and Flutaxl-loaded nanoparticles released their payload with different kinetic profiles, as indicated by changes in the relaxation times (Supplementary Fig. 7c-f). Specifically, Doxorubicin was completely released within 4 days, which resulted in restoration of relaxation times (Supplementary Fig. 7c,d). Flutax1 demonstrated sustained release in serum during the 10-day duration of the study with $\sim 50 \%$ of the drug released within 7 days (Supplementary Fig. 7e,f). We attribute these release patterns to the different chemical characteristics of each payload. Doxorubicin may be quickly released, due to lower hydrogen bonding formation between its amino end and the polymer's carboxyl and hydroxyl groups that results in its overall weaker association with the nanoparticles. On the other hand, Flutaxl has multiple carbonyl and methyl groups that facilitate its tighter association with Ferumoxytol coating, as well as multiple segments that favour hydrophobic interactions.

Intracellular cargo release de-quenches the nanophores. We next investigated whether cargo-loaded nanophores could deliver drugs to cancer cells in vitro. Incubation of the prostate cancer
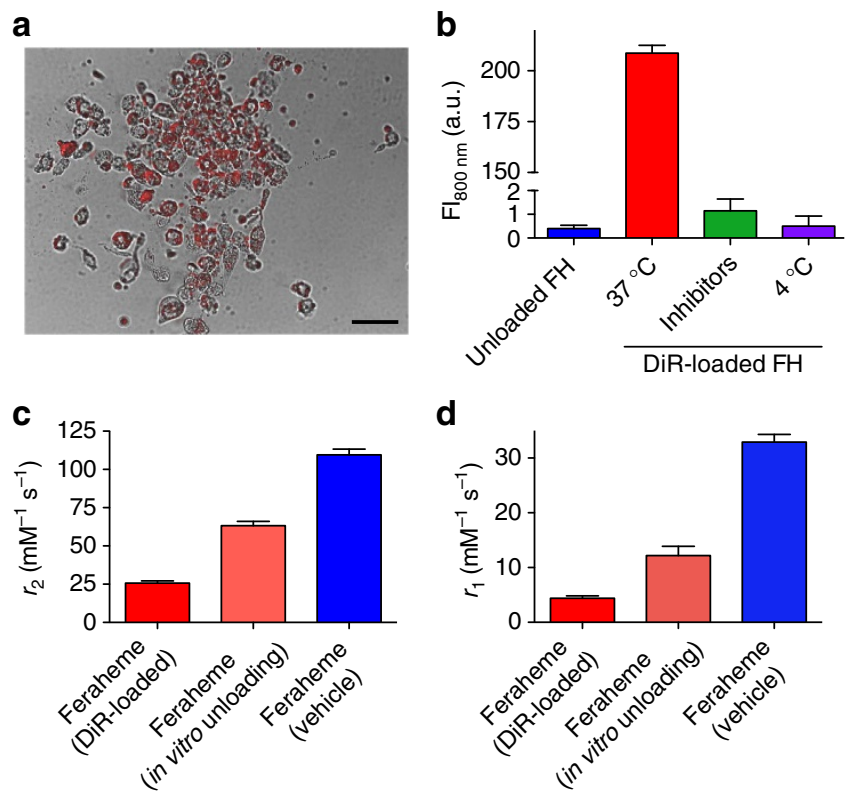

Figure 4 | Intracellular cargo delivery with nanophores. (a) Composite image of bright field and fluorescence images of LNCaP cells treated for $48 \mathrm{~h}$ with Doxorubicin-loaded Ferumoxytol. Doxorubicin fluorescence is shown in red (scale bar, $50 \mu \mathrm{m}$ ). (b) Cells were treated with DiR-loaded Ferumoxytol, and after washing and trypsinization, the harvested cell pellets were imaged with an Odyssey reader at $800 \mathrm{~nm}$ to quantify the uptake of the nanophores. Control cells were treated with unloaded nanoparticles. Inhibition of endocytosis was performed at $4{ }^{\circ} \mathrm{C}$ and with the inhibitors sodium azide and 2-deoxyglucose (mean \pm s.e.m., $n=3$ ). The cell pellets were subjected to iron digestion, and revealed that upon release of the cargo the $r_{2}$ (c) and $r_{1}$ (d) relaxivities of DiR-carrying Ferumoxytol were higher than those of the corresponding fully loaded formulation (mean \pm s.e.m., $n=3$ ).

cell line LNCaP with Doxorubicin-loaded Ferumoxytol for $48 \mathrm{~h}$ resulted in significant drug uptake, as indicated by the enhanced cellular fluorescence due to the presence of Doxorubicin (Fig. 4a). Similarly, high cell-associated fluorescence was seen in LNCaP cells incubated for $48 \mathrm{~h}$ with nanophores carrying the nearinfrared fluorophore DiR, compared with empty control nanoparticles (Fig. 4b). Inhibition of the endocytic process with sodium azide and 2-deoxyglucose or incubation at $4{ }^{\circ} \mathrm{C}$ prevented Ferumoxytol's uptake and led to nominal fluorescence, which demonstrated that Ferumoxytol was taken up via the functional endocytic machinery and not through passive means. In addition, we found that the unloading of DiR within the cells recovered Ferumoxytol's superparamagnetic properties, which approached the $r_{2}$ and $r_{1}$ relaxivities of the parent empty nanoparticles (Fig. 4c,d). It is likely that some of the cargo might have still been retained within Ferumoxytol, consequently affecting its properties and preventing $r_{2}$ and $r_{1}$ to fully regain the relaxivity of the unloaded nanoparticles. Since Ferumoxytol undergoes lysosomal degradation with release of iron cations, the observed changes in relaxivity are most likely attributed to cargo release, and not due to nanoparticle aggregation within the endocytic vesicles. Our findings demonstrate that measurement of nanoparticle relaxation can be used for the sensitive characterization of non-fluorescent payloads carried by IONP utilizing portable relaxometers ${ }^{33,34}$, NMR analysers and MRI.

The cargo affects nanophores' water accessibility. To elucidate the cargo's effect on the relaxivity, we reasoned that once the payload intercalates non-covalently within the nanophores' 
polymeric coating, it might obstruct the free diffusion of water to the vicinity of their magnetic core. This may reduce the nanoparticles' capability to affect the relaxation time of the bulk water and consequently result in higher relaxation times (Fig. 5a). To study whether the cargo limits the accessibility of water molecules or exerts an effect by itself, we evaluated DiR-loaded IONP in increasing concentrations of deuterium oxide $\left(\mathrm{D}_{2} \mathrm{O}\right)$ instead of $\mathrm{H}_{2} \mathrm{O}$. Deuterium has a different magnetic moment from hydrogen, allowing its use in the identification of the role of water and its hydrogen protons via NMR methods. If the drug would exert an intrinsic effect then the changes in relaxivity would not be affected as much by the Deuterium. However, with increasing amount of $\mathrm{D}_{2} \mathrm{O}$, the $\mathrm{T} 2$ and $\mathrm{T} 1$ signal of DiR-loaded IONP solutions decreased, indicating that the changes in relaxation times are due to the cargo reducing the access of the bulk water molecules rather than an effect exerted by the drug itself (Fig. 5b,c; Supplementary Fig. 8a,b). Since the relaxation of water by IONP arises from the water molecules diffusing near the nanoparticles ${ }^{35,36}$, we utilized diffusion-weighted MRI, in order to confirm with an additional method that the cargo impairs the diffusion of water molecules within the nanophores' coating. The apparent diffusion coefficient map revealed that the Flutax1- and Doxorubicin-loaded Ferumoxytol had lower diffusion coefficients than the unloaded nanoparticles (vehicle) at the same particle concentration (Fig. 5d-f). This supported our hypothesis that the changes in relaxation times are due to the cargo reducing the diffusion of water within the nanoparticles rather than the cargo itself exerting a direct effect.

Previous studies have utilized the target-induced clustering of IONP in sensitive assays for the detection of numerous biomolecules and targets $24,29,30$. Specifically, it was demonstrated by others and us that the nanoparticles form extensive supramolecular assemblies in the presence of their target ${ }^{30,37,38}$. The formation of these assemblies containing multiple nanoparticles was predominantly associated with T2 decreases but no reported effect on T1 (ref. 39). While we did not observe any size changes to indicate clustering (Fig. 1c; Supplementary Fig. 1a), we nevertheless sought to confirm that the mechanism described herein is not due to aggregation-induced changes. We utilized Concanavalin A (Con A), a protein with high affinity towards carbohydrates ${ }^{40,41}$, to facilitate the clustering of the carbohydrate-coated (carboxymethyl dextran) Ferumoxytol. As expected, addition of the Con A to Ferumoxytol induced decrease in the solution's T2 but little increase in the T1 (Supplementary Fig. 9a,b), with nanoparticle aggregation confirmed with DLS (Supplementary Fig. 9c). Moreover, when excess dextran was used to obtain larger clusters ([Dextran] $=2.5 \mathrm{mg} \mathrm{ml}^{-1}$ ), the T1 increased, but the T2 decreased (Supplementary Fig. 9a-c) due to Ferumoxytol's Con A-induced clustering and did not increase as seen secondary to drug loading. Therefore, these results demonstrate that the effect of cargo on the nanoparticles, such as after drug loading, is novel, and not based on nanoparticle

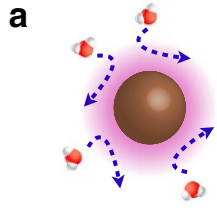

b

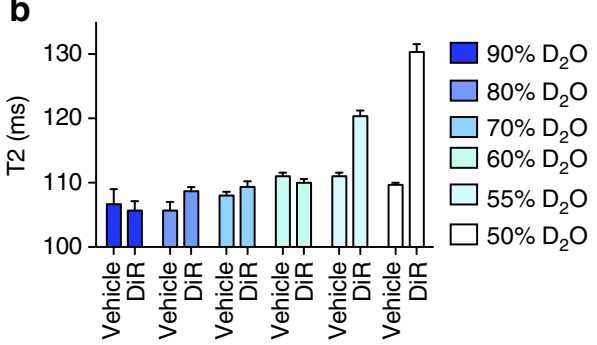$$
\text { d }
$$

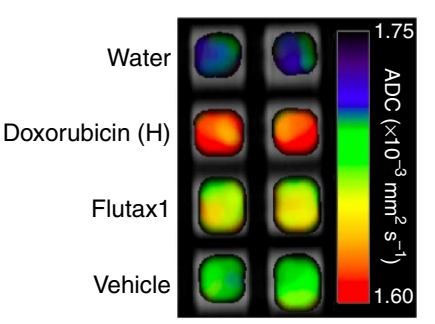

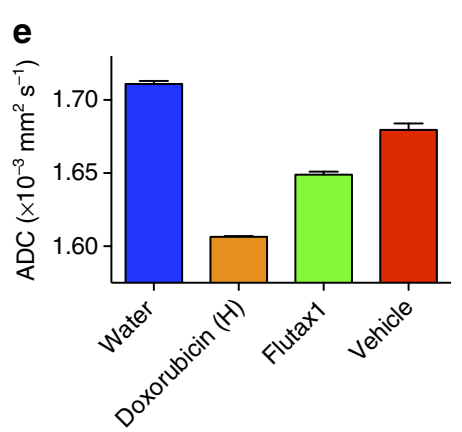

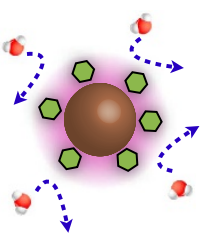

C

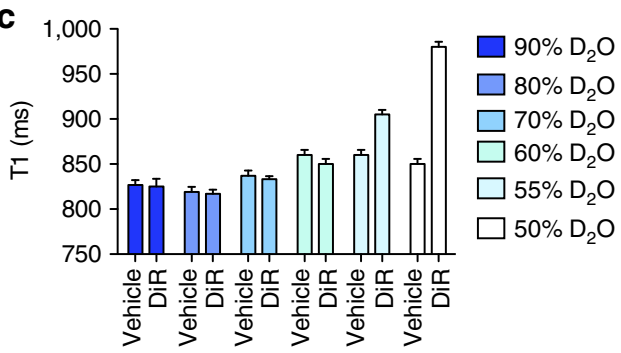

f

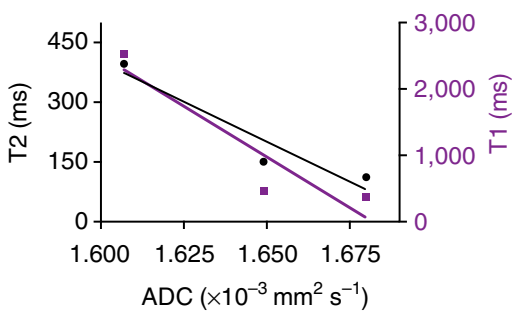

Figure 5 | The cargo obstructs the access of water in the nanophores' proximity. (a) Schematic representation of the proposed model that suggests that the presence of cargo within the coating of IONP hinders the diffusion of water molecules, concomitantly affecting the ability of nanoparticles to efficiently dephase water's protons. At high $\mathrm{D}_{2} \mathrm{O}$ concentrations, the changes on (b) T2 and (c) T1 were abrogated (mean \pm s.e.m., $n=3$ ), suggesting that the observed increases in T2 and T1 during cargo loading occurred upon blockage of water molecules by the entrapped cargo ([Fe $]_{\text {PAA-IONP }}=2.5 \mu \mathrm{gl} \mathrm{ml}^{-1}$ ) rather than an effect exerted by the payload. (d-f) Diffusion-weighted MRI revealed that the presence of molecular payload within Ferumoxytol's coating affected the diffusion of water molecules ( $[\mathrm{Fe}]_{\text {Ferumoxytol }}=5 \mu \mathrm{g} \mathrm{ml} \mathrm{l}^{-1}$ for all wells; mean \pm s.e.m., $n=6$ ). The cargo's effect on ADC correlated with the observed changes in T2 and T1 signal (mean \pm s.e.m., $n=6$; linear regression correlation coefficients $r_{\mathrm{T} 2}=0.95$ and $r_{\mathrm{T} 1}=0.92$; vehicle: unloaded nanoparticles; mean \pm s.e.). 
clustering. This likely explains why previous studies have not identified the effect of clustering on T1 (ref. 39), as the extent of the aggregation state is critical.

Improved therapy efficacy with cargo-carrying nanophores. Finally, we examined whether Ferumoxytol-based nanophores could efficiently deliver chemotherapeutics to cancer cells and cause cytotoxicity. Due to the aberrant signalling of many pathways in cancer, combinatorial therapies ideally require the successful administration of several chemotherapeutics at the same time. Towards this direction, we utilized Ferumoxytol as a drug delivery vehicle for combinatorial therapy in prostate cancer, in order to concurrently inhibit the crosstalk between the androgen receptor pathway and the PI3K cascade and prevent resistance to chemotherapy ${ }^{32}$. We simultaneously encapsulated BEZ235-a PI3K inhibitor-together with the androgen receptor antagonist MDV3100 in order to deliver both drugs to prostate cancer cells. Dose-response studies revealed that nanophores loaded with both BEZ235 and MDV3100 had an IC50 of $10 \mathrm{nM}$ (BEZ235) and $0.2 \mu \mathrm{M}$ (MDV3100) for the human prostate adenocarcinoma cells LNCaP cultured in media with charcoal-stripped serum, where the serum was depleted of any androgens (Fig. 6a). However, administration of both drugs in their free forms had IC50 values of $75 \mathrm{nM}$ (BEZ235) and $1.5 \mu \mathrm{M}$ (MDV3100). The unloaded nanophores did not affect the cells' viability, which demonstrated that the drug delivery vehicle lacked any intrinsic cytotoxic properties. To confirm that the nanophores could facilitate the cargo's tumour delivery, we used nanophores loaded with the near-infrared fluorophore DiR and performed reflectance fluorescence imaging. Twenty-four hours after injection, there was enhanced fluorescence in the tumours, showing that the nanophores targeted to the tumours with no observable signal from other organs on surface weighted fluorescence imaging (Fig. 6b). In vivo therapy studies with nude male mice bearing PC3-derived prostate cancer xenografts (Fig. 6c,d) and nude female mice with breast cancer BT20 tumours (Fig. 6e) revealed that the drug-loaded nanophores were considerably more effective than the free drugs, following intravenous (i.v.) administration. Notably, delivery of bortezomib with nanophores achieved enhanced tumour regression, while the free drug was ineffective and marginally suppressing tumour growth when compared with the tumour's volume of control (DMSO-treated) animals (Fig. 6f). Prostate and breast tumours treated with doxorubicin-carrying nanoparticles significantly regressed, as opposed to the free drug that only achieved tumour control at its initial pre-treatment size and vehicle (DMSO) therapy that resulted in continuous tumour growth (Fig. $6 \mathrm{~g}, \mathrm{~h}$ ).

Biodistribution studies with radiolabeled PU-H71 ( ${ }^{131} \mathrm{I}-\mathrm{PU}$ H71) showed that the nanophores substantially improved the delivery of the drug to the tumours, serving as efficient, longcirculating delivery vehicles (Fig. 6i,j). We selected this Hsp90 inhibitor for these studies, because this drug contains as iodine atom, which can be substituted with radioactive iodine, such as ${ }^{131}$ I, without altering the drug's structure and intermolecular interactions. Twenty-four hours post i.v. administration, the amount of drug at the tumours more than doubled thanks to the nanophore-based delivery. Apart from higher tumour uptake, the nanophores allowed the drug to stay longer in circulation, as indicated by the higher levels of radiolabeled compound in blood, lungs and spleen. This allows a larger amount of chemotherapeutic to be released at the tumour, without the need for higher dosages or more frequent drug administration. These results also suggested that the nanophores are cleared through the hepatic route, similar to other nanoparticles of the same size. Taken together, these findings demonstrate that encapsulation of drugs within the nanophores enhances the therapeutics' bioavailability, preventing their nonspecific association with proteins and lipids, while delivering them within tumours and cells at effective dosages, thus vastly improving the efficacy of the utilized drugs over their free administration.

Monitoring drug release in vivo with MRI. Finally, monitoring of in vivo drug release was achieved by examining changes in the $\mathrm{T} 2$ and $\mathrm{T}^{\star}$ relaxation times via MRI (Fig. $7 \mathrm{a}-\mathrm{d}$ ). Our imaging studies showed that 2 and $4 \mathrm{~h}$ after i.v. administration, the tumour T2 signal of animals treated with drug-loaded FH was higher than that of mice treated with empty nanoparticles (Fig. 7a,c). At the same time, there were no significant differences in the tumour $\mathrm{T} 2^{\star}$ of animals treated with drug-loaded or empty nanoparticles, indicating similar particle concentrations in the tumour. However, as expected, the presence of the nanophores at the tumour caused decrease in $\mathrm{T}^{\star}$ compared with the pre-administration $(0 \mathrm{~h})$ reading (Fig. $7 \mathrm{~b}, \mathrm{~d})$. Twenty-four hours after nanophore administration, there were no differences between the tumour $\mathrm{T} 2$ and $\mathrm{T}^{*}$ of animals treated with Doxorubicin-loaded Ferumoxytol or empty nanophores, demonstrating that release of the drug occurred in vivo due to the vehicle's fast drug release kinetics (Figs 1e,f and 3a,b).

\section{Discussion}

Here, we demonstrated the use of clinical nanophores as drug carriers, in a process that relies on weak electrostatic interactions and preserves both the drug's and nanoparticle's structure, while enhancing their aqueous stability and bioavailability. We showed that Ferumoxytol is a diverse drug delivery platform, accommodating payloads with a wide range of molecular weights. As the cargo incorporation causes significant changes in the nanophores' magnetic properties, the loading of non-fluorescent agents-otherwise difficult to monitor-can be evaluated via magnetic relaxation, thus providing a drug delivery platform that self-reports the drug delivery and release through the changes in their MR signal properties. Importantly, the therapeutic efficacy was significantly improved over the free drug using nanophores. Therefore, this novel integrated drug delivery and monitoring strategy, which employs clinically approved agents in a nonaltered form, is suitable to improve chemotherapy of cancer significantly and will be an integral part of understanding the dynamics, risk assessment and approval of nanoparticle-based drug delivery ${ }^{12}$.

\section{Methods}

Materials. All chemicals were of analytical grade, unless otherwise stated. Ferrous and ferric chloride $\left(\mathrm{FeCl}_{2} \cdot 4 \mathrm{H}_{2} \mathrm{O}\right.$ and $\left.\mathrm{FeCl}_{3} \cdot 6 \mathrm{H}_{2} \mathrm{O}\right)$ were from Fluka and $\mathrm{D}_{2} \mathrm{O}$ was from Acros. PAA (MW $1.8 \mathrm{kDa}$ ), ammonium hydroxide, hydrochloric acid and dimethyl sulfoxide (DMSO) were from Sigma-Aldrich. Dextran $(10 \mathrm{kDa})$ was acquired from Pharmacosmos, while Con A was bought from Sigma-Aldrich. Payload included the following compounds: Alendronate (MW: 325) from SigmaAldrich, AZD8055 (MW: 466) from Selleck Chemicals, BEZ235 (MW: 470) from Cayman Chemicals, BKM120 (MW: 580) was a gift from Professor Lewis Cantley (Weill Cornell Medical College, Cornell University), Dasatinib (MW: 488) from Selleck Chemicals, DiR (1,1'-dioctadecyl-3,3,3',3'-tetramethylindo-tricarbocyanine iodide, MW: 1,013) from Invitrogen, doxorubicin (Adriamycin, MW: 580) from Selleck Chemicals, Flutax1-a fluorescent Taxol analogue (MW:1337) - from Tocris Bioscience, FR230 (MW: 687) was provided by Dr Horst Kessler (Technische Universität München), GSI-34 (MW: 534) was provided by

Dr Yueming Li (MSKCC), Lapatinib ditosylate (MW: 925) was purchased from Selleck Chemicals, MDV3100 (MW: 464) was provided by Professor Charles Sawyers (MSKCC), Bortezomib (MW: 384) was purchased from Selleck Chemicals and PU-H71 (MW: 512) was provided by Dr Gabriela Chiosis (MSKCC). Stocks of these chemicals were prepared in DMSO, and stored at $-20^{\circ} \mathrm{C}$ according to the suppliers' instructions. Commercial IONP preparations were obtained from Micromod Partikeltechnologie GmbH (Rostock, Germany; $\mathrm{NH}_{2}$-nanomag-D-spio) and AMAG Pharmaceuticals (Lexington, MA; Ferumoxytol). 
a

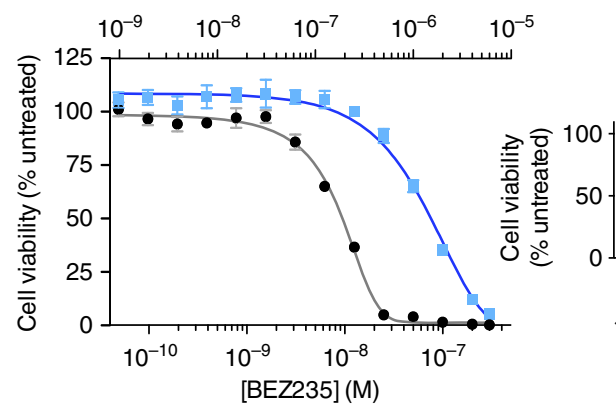

C

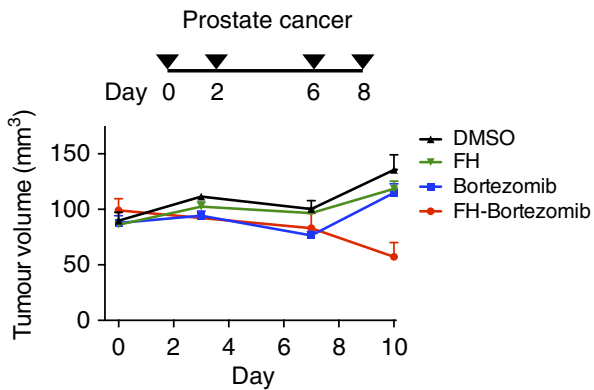

f

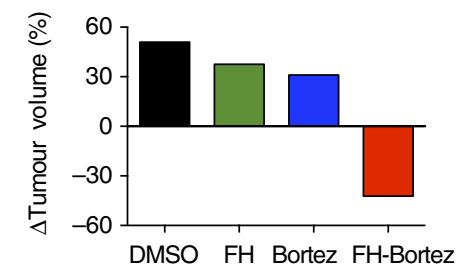

b

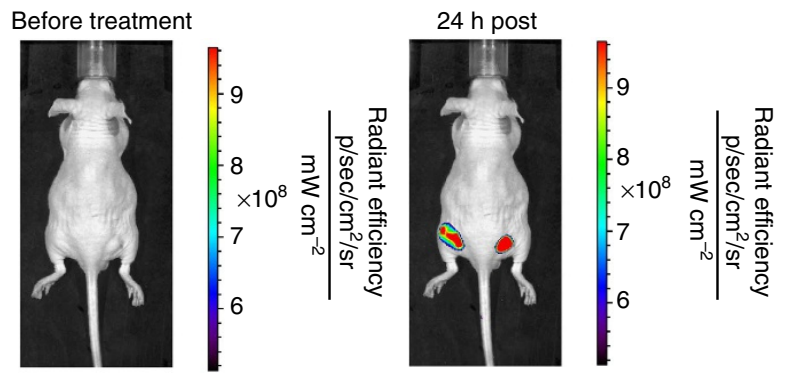

d
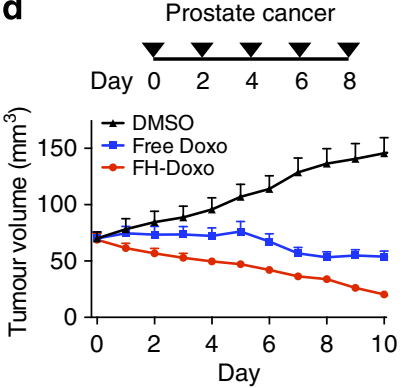

$\mathbf{g}$

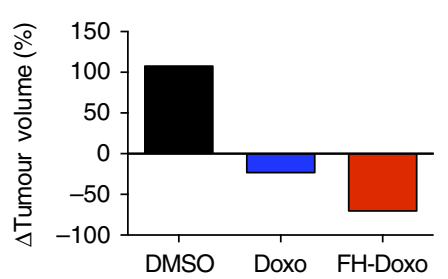

e
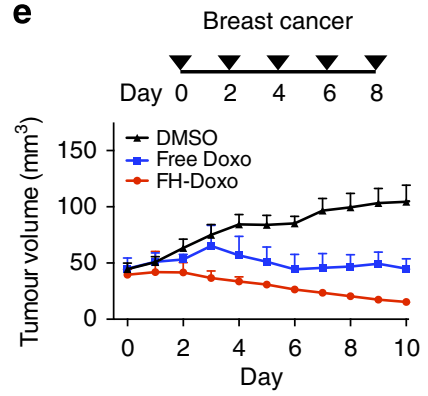

h

Breast cancer

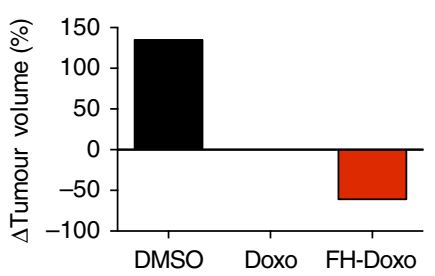

i $24 \mathrm{~h}$ j Tumour uptake

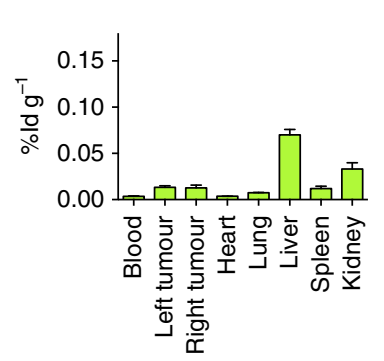

PU-H71

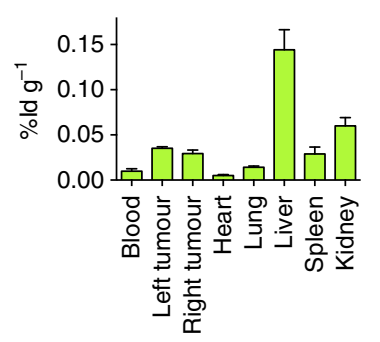

FH-PU-H71

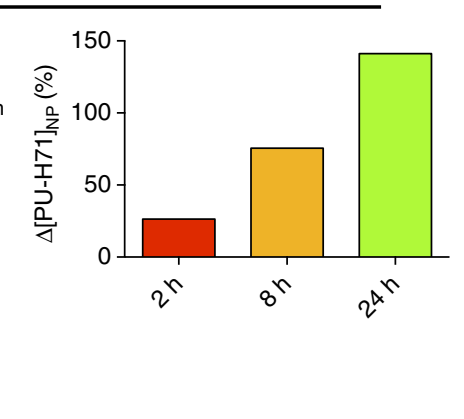

Figure 6 | Nanophores as in vitroand in vivo chemotherapeutic vehicles. (a) Cytotoxicity profile of the human prostate cancer cells LNCaP treated with co-administered free drugs or Ferumoxytol that was loaded with both the PI3 kinase inhibitor BEZ235 and the anti-androgen MDV3100 (mean \pm s.e.m., $n=3$ ). (b) Representative IVIS images of DiR-loaded nanophores demonstrating the fluorophore's localization in the tumours. (c-e) Drug-loaded nanophores (FH-Bortezomib or FH-Doxo) efficiently reduced tumour volume in mice bearing (c,d) human prostate and (e) human breast xenografts (mean \pm s.e.m.; $n=3$ per treatment group for the Bortezomib study; for prostate cancer chemotherapy with Doxorubicin: $n_{\mathrm{DMSO}}=3, n_{\mathrm{Doxo}}=3, n_{\mathrm{FH}-\mathrm{Doxo}}=4$; for breast cancer chemotherapy with Doxorubicin: $\left.n_{\mathrm{DMSO}}=3, n_{\mathrm{DoxO}}=3, n_{\mathrm{FH}-\mathrm{Doxo}}=4\right)$. $(\mathbf{f}-\mathbf{h})$ The bar graphs depict the change in tumour volume between day 10 and 0 of the $(\mathbf{c}, \mathbf{d})$ treatment regimes. (i) Biodistribution profiles of the free and nanophore-encapsulated ${ }^{131} \mathrm{I}-\mathrm{PU}-\mathrm{H} 7124 \mathrm{~h}$ after administration ( $n=4$ per treatment group). (j) Tumour retention profiles of free and nanophore-encapsulated ${ }^{131} \mathrm{I}-\mathrm{PU}$-H71 (\%ld/g: \% injected dose/tissue mass, $n_{2 h}=3$ per treatment group, $n_{8 h}=3$ per treatment group, $n_{24 h}=4$ per treatment group), with the corresponding net change in drug delivery and retention achieved with the nanophores $\left(\Delta[\mathrm{PU}-\mathrm{H} 71]_{\mathrm{NP}}\right)$.

In-house preparation of alternate IONP. PAA-coated IONP were prepared with the alkaline precipitation method, involving the rapid mixing of ferrous and ferric chloride in ammonium hydroxide that was followed by addition of the polymer solution $^{42}$. To remove excess reagents and byproducts, the nanoparticles were washed, concentrated and reconstituted in pH 7.4 PBS, with a KrosFlo Research II TFF system that was equipped with a $10 \mathrm{kDa}$ column (Spectrum). The nanoparticles were stored at $4{ }^{\circ} \mathrm{C}$ until further use, without any precipitation being observed, similar to the aminated nanoparticles obtained from Micromod, which were used without any additional preparation. Ferumoxytol was subjected to magnetic separation using a $1 \times$ PBS-equilibrated LS25 MACS column (Miltenyi, Cambridge, MA), in order to isolate IONP with good magnetic properties from any free polymer in the solution. Subsequently, Ferumoxytol was stored at $4^{\circ} \mathrm{C}$ until further use.

Drug loading into nanophores. Encapsulation of the molecular payload was achieved using a modified solvent-diffusion-based protocol, facilitating the 
a

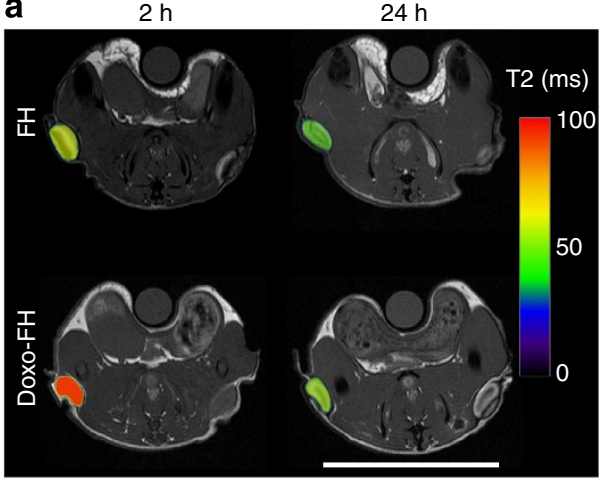

C 100

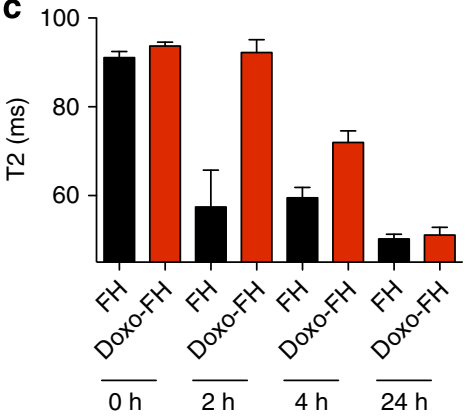

b

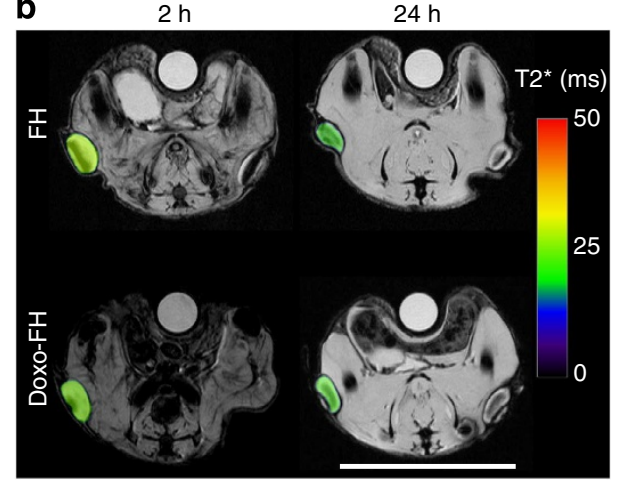

d

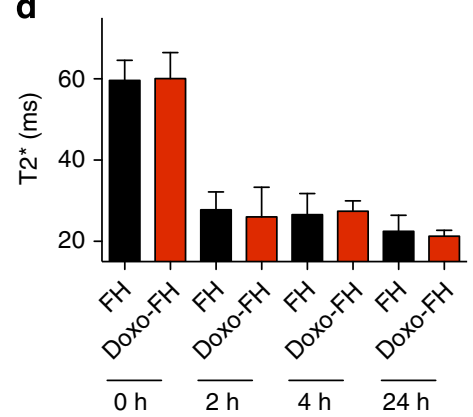

Figure 7 | Noninvasive monitoring of nanophore drug release in vivo with MRI. Mice were injected with unloaded (FH) or Doxorubicin-loaded Ferumoxytol (Doxo-FH), and the tumours (a) T2 and (b) T2* signals were monitored across time (scale bars, 25 mm). (c) As Ferumoxytol released doxorubicin in vivo, the nanoparticles' T2 signal gradually decreased and eventually reached that of the unloaded nanoparticles (mean \pm s.e.m.; $n=3$ per treatment group per time point). (d) The T2* indicated that the unloaded and loaded nanoparticles were equally retained at the tumours, as there were no differences in the $\mathrm{T}^{\star}{ }^{\star}$ signal (mean \pm s.e.m.; $n=3$ per treatment group per time point).

entrapment of hydrophobic molecules within IONP's polymeric coating. In general, the nanoparticles $\left(25 \mu \mathrm{l}\right.$ of either PAA-IONP or $\mathrm{NH}_{2}$-nanomag-D-spio, $30 \mu \mathrm{l}$ of Ferumoxytol) were resuspended in $500 \mu \mathrm{l}$ distilled water, whereas the cargo was diluted to the desired concentration in DMSO (final volume of payload solution was $100 \mu \mathrm{l})$. The fluorophore or drug payload solution was added dropwise to the nanoparticle solution under vortexing (1,000 r.p.m.) at room temperature, without any visible precipitation. Subsequently, the preparation was subjected to dialysis in a small-volume dialysis chamber (MWCO 3000, Fisher) against $1 \times$ PBS. The cargo-carrying IONP were subsequently stored in the dark at $4^{\circ} \mathrm{C}$, until further use.

Nanophore characterization. The size of the nanophores was determined through DLS (Nano-ZS, Malvern, Westborough, MA). The same instrument was used in nanoparticle surface charge measurement ( $\zeta$ potential), whereas to determine Ferumoxytol's nanoparticle concentration the NS500 instrument was utilized (NanoSight, Duxbury, MA). Magnetic relaxation measurements, including $r_{1}$ and $r_{2}$ relaxivities, were determined with a $0.47 \mathrm{~T}$ mq20 NMR analyser (Minispec, Bruker, Billerica, MA). For T2 measurements, a CPMG pulse-echo train with a $1.5 \mathrm{~ms}$ interpulse spacing was used, whereas the T1 sequence varied the interpulse spacing from $5 \mathrm{~ms}$ up to $8,500 \mathrm{~ms}$. The preparations' iron concentration was determined spectrophotometrically as previously reported ${ }^{43}$, using a SpectraMax M5 instrument (Molecular Devices, Sunnyvale, CA). Briefly, the nanoparticles were subjected to acid digestion, and subsequent conversion of all iron ions to iron(III). A standard curve was created based on the absorbance at $410 \mathrm{~nm}$ of solutions of known concentration of $\mathrm{FeCl}_{3}$ in the digesting solution. Fluorescence emission measurements were performed using the SpectraMax M5, as well as an Odyssey near-infrared imaging station (LI-COR Biosciences, Lincoln, NE), equipped with two solid-state lasers for excitation at 685 and $785 \mathrm{~nm}$. To determine the cargo load of each preparation, the following molar extinction coefficients were used: $\varepsilon_{\text {Doxorubicin }}=11,500 \mathrm{M}^{-1} \mathrm{~cm}^{-1}$ at $480 \mathrm{~nm}, \varepsilon_{\mathrm{Flutax1}}=52,000 \mathrm{M}^{-1} \mathrm{~cm}^{-1}$ at $495 \mathrm{~nm}$ and $\varepsilon_{\text {DiR }}=270,000 \mathrm{M}^{-1} \mathrm{~cm}^{-1}$ at $748 \mathrm{~nm}$. For all other cargo, we quantified the amount of drug-loaded into the nanoparticles using HPLC and standard curves with known amounts of the corresponding drug. We first induced release of the cargo by incubating the loaded nanoparticles in a $2 \mathrm{M} \mathrm{NaCl}$ solution for $30 \mathrm{~min}$, followed by spin filtration (MWCO 5000) to collect the cargocontaining solution. Stability experiments were performed in $\mathrm{pH}$-adjusted $\mathrm{PBS}$, whereas serum experiments were performed at $37^{\circ} \mathrm{C}$, using fetal bovine serum obtained from Gemini Bio-products. The sterile serum lacking any nanoparticles had a T2 of $600 \pm 10 \mathrm{~ms}$ and T1 of $1,700 \pm 30 \mathrm{~ms}$ (mean \pm s.e.m.; $n=3$ ), which remained unaltered during the experiments. The clustering of Ferumoxytol
$\left([\mathrm{Fe}]=20 \mu \mathrm{g} \mathrm{ml}^{-1}\right)$ was studied in the presence of Con A $\left([\text { Con A }]_{\text {final }}=\right.$ $50 \mu \mathrm{g} \mathrm{ml}^{-1}$ ) using DLS and the Minispec. Release of doxorubicin from drugloaded Ferumoxytol was performed using a dynamic dialysis setup, as previously described $^{22}$. A dialysis chamber was utilized (MWCO 3000, Fisher), containing doxorubicin-loaded Ferumoxytol in either $\mathrm{pH} 7.2$ or $\mathrm{pH} 6.81 \times$ PBS. The nanoparticles were dialyzed against the corresponding $\mathrm{pH}$-adjusted buffer at room temperature and under constant stirring ( 150 r.p.m.), where at regular time intervals, aliquots from the external aqueous milieu of the device were collected for further analysis. The collected samples were analysed via a Beckman Coulter HPLC instrument, equipped with a $\mathrm{C} 18$ reverse phase column and set to monitor doxorubicin's absorbance at $480 \mathrm{~nm}$ mixing An animal MRI from Bruker Biospin (Billerica, MA) operating at $4.7 \mathrm{~T}$ and a $35-\mathrm{mm}$ radiofrequency coil were used to image phantoms of the nanoparticle preparations that were spotted on a microplate.

In vitro drug release from loaded nanophores. $\mathrm{LNCaP}$ cells were grown to confluence, on a 12-well poly(lysine)-coated plate in 10\% FBS-containing RPMI medium at $37^{\circ} \mathrm{C}, 5 \% \mathrm{CO}_{2}$. The medium was aspirated, and the cells were supplemented with $1 \mathrm{ml}$ fresh media, plus $50 \mu \mathrm{l}$ of either empty (vehicle), Doxorubicin-loaded Ferumoxytol or DiR-loaded Ferumoxytol. After $48 \mathrm{~h}$, the cells treated with Doxorubicin-loaded Ferumoxytol were examined under a Nikon Eclipse TiE fluorescence microscope, in order to determine the nanoparticle uptake. Likewise, following $48 \mathrm{~h}$-long incubation at $37^{\circ} \mathrm{C}, 5 \% \mathrm{CO}_{2}$, the cells treated with vehicle and DiR-loaded nanoparticles were trypsinized and subjected to centrifugation at 1,000 r.p.m. for $6 \mathrm{~min}$. The resulting pellets were then resuspended in $400 \mu \mathrm{l} 1 \times$ PBS and aliquoted in two eppendorf tubes for fluorescence emission and magnetic relaxation measurements, using the nearinfrared imager (LI-COR) and the benchtop relaxometer (Bruker). Studies of inhibition of nanoparticle uptake were performed at $37^{\circ} \mathrm{C}, 5 \% \mathrm{CO}_{2}$ in the presence of sodium azide $(10 \mathrm{mM})$ and 2-deoxyglucose $(50 \mathrm{mM})$, as well as at $4{ }^{\circ} \mathrm{C}$, with either way inhibiting active endocytosis. For near-infrared fluorescence, excitation was achieved at $785 \mathrm{~nm}$, with emission recorded at $800 \mathrm{~nm}$; with the instrument settings set as follows: focus offset $=4 \mathrm{~mm}$, intensity $=0.5$ and resolution $=169$ $\mu \mathrm{m}$. The iron content of the cell pellets was determined as described above with untreated samples of equal cell numbers serving as control.

Cell viability and in vivo studies. $\mathrm{LNCaP}, \mathrm{PC} 3$ and $\mathrm{BT} 20$ cells were obtained from ATCC (Manassas, VA), and maintained according to the supplier's instructions. LNCaP cells were seeded on black-walled, clear bottom 96-well plates at a cell 
density of 10,000 cells per well, supplemented with $100 \mu \mathrm{l} 10 \%$ FBS-containing RPMI medium. Controls included cells incubated with unloaded nanoparticles or DMSO, corresponding to the free drug's final solvent concentration. Dose-response curves were obtained after the cells were treated for $48 \mathrm{~h}$ with corresponding agent. Subsequently, the old medium was aspirated, and cell viability was assessed via the Alamar Blue method (Invitrogen). Briefly, the cells were supplemented with $10 \%$ Alamar blue-containing medium (10\% FBS-containing RPMI), followed by 3-h incubation in a humidified incubator $\left(37^{\circ} \mathrm{C}, 5 \% \mathrm{CO}_{2}\right)$ and recording of fluorescence emission $\left(\lambda_{\mathrm{exc}}=565 \mathrm{~nm}, \lambda_{\mathrm{em}}=585 \mathrm{~nm}\right)$ with the SpectraMax M5 plate reader. The localization of DiR-loaded nanophores was assessed with an IVIS 200 in vivo imaging system, equipped with an ICG filter set (Waltham, MA). Adult, male, nude mice $(n=3)$ with LNCaP xenografts on their flanks were administered $100 \mu \mathrm{l}$ of nanophores $([\mathrm{DiR}]=400 \mu \mathrm{M})$ i.v. (radiant efficiency $\max _{\max }=9.1 \pm 0.4 \times 10^{8}$; mean \pm s.e.m.). Adult, male, nude mice $(n=12)$ that had PC 3 tumours on their flanks were treated on days $0,2,6$ and 8 with $100 \mu \mathrm{l}$ of equimolar ([Bortezomib] $=0.5 \mathrm{mM}$ ) concentrations of either free (diluted in 5\% DMSOcontaining 1X PBS) or Ferumoxytol-encapsulated bortezomib. Control animals were treated with either 5\% DMSO-containing 1X PBS or unloaded Ferumoxytol that had the same iron concentration as the loaded nanophores $([\mathrm{Fe}]=0.75$ $\left.\mathrm{mg} \mathrm{ml}^{-1}\right)$. Adult, male, nude mice $(n=10)$ bearing PC3 tumours and adult, female, nude mice $(n=10)$ with BT20 tumours on their flanks were treated every other day (days $0,2,4,6$ and 8) with $100 \mu$ ( (retroorbital injection) of either doxorubicin alone or doxorubicin-loaded Ferumoxytol, both at a final doxorubicin concentration of $0.28 \mathrm{mM}$. Control animals were treated with $100 \mu \mathrm{l}$ of $10 \%$ DMSO-containing $1 \times$ PBS to match the DMSO content of free Doxorubicin, since the drug was dissolved in DMSO and diluted to the desired concentration in PBS. Change in tumour volume was defined as the ratio of the tumour volume on day 10 minus the tumour volume on day 0 divided by the tumour volume of day 0 . For biodistribution studies, we used 20 adult, male, nude mice with PC3 xenografts on their flanks, and either free or Ferumoxytol-loaded ${ }^{131}$ I-PU-H71. Following retroorbital administration, the mice were euthanized at the designated time points, and the radioactivity of the collected organs was measured on PerkinElmer's (Waltham, MA) Wizard ${ }^{2} 2480$ Automatic Gamma Counter. Change in drug uptake and retention following nanophore administration $\left(\Delta[\mathrm{PU}-\mathrm{H} 71]_{\mathrm{NP}}\right)$ was calculated as the ratio of nanoparticle-delivered radiolabeled drug minus the free radiolabeled drug divided by the free radiolabeled drug. An animal MRI from Bruker Biospin (Billerica, MA) operating at $4.7 \mathrm{~T}$ and a $35-\mathrm{mm}$ radiofrequency coil was used to image the mice. Changes in tumour size were evaluated with a microcaliper, and at the end of the study, the mice were euthanized, according to the MSKCC Institutional Animal Care and Use Committee guidelines.

Data analysis. All experiments were performed in triplicate unless otherwise stated, with the results presented as mean \pm s.e.m. The data were analysed in Prism (GraphPad Software), whereas the MR images were processed through the OsiriX DICOM viewer.

\section{References}

1. Duncan, R. The dawning era of polymer therapeutics. Nat. Rev. Drug Discov. 2, 347-360 (2003).

2. Duncan, R. Polymer conjugates as anticancer nanomedicines. Nat. Rev. Cancer 6, 688-701 (2006)

3. Huang, P. S. \& Oliff, A. Drug-targeting strategies in cancer therapy. Curr. Opin. Genet. Dev. 11, 104-110 (2001)

4. Moses, M. A., Brem, H. \& Langer, R. Advancing the field of drug delivery: taking aim at cancer. Cancer Cell 4, 337-341 (2003).

5. Qian, Z. M., Li, H., Sun, H. \& Ho, K. Targeted drug delivery via the transferrin receptor-mediated endocytosis pathway. Pharmacol. Rev. 54, 561-587 (2002).

6. Kularatne, S. A., Wang, K., Santhapuram, H. K. \& Low, P. S. Prostate-specific membrane antigen targeted imaging and therapy of prostate cancer using a PSMA inhibitor as a homing ligand. Mol. Pharm. 6, 780-789 (2009).

7. Low, P. S., Henne, W. A. \& Doorneweerd, D. D. Discovery and development of folic-acid-based receptor targeting for imaging and therapy of cancer and inflammatory diseases. Acc. Chem. Res. 41, 120-129 (2008).

8. Gaind, V., Kularatne, S., Low, P. S. \& Webb, K. J. Deep-tissue imaging of intramolecular fluorescence resonance energy-transfer parameters. Opt. Lett. 35, 1314-1316 (2010).

9. Lu, Z. R. Molecular imaging of HPMA copolymers: visualizing drug delivery in cell, mouse and man. Adv. Drug Deliv. Rev. 62, 246-257 (2010).

10. Theeraladanon, C. et al. Rational approach to the synthesis, evaluation, and (68)ga labeling of a novel 4-anilinoquinoline epidermal growth factor receptor inhibitor as a new imaging agent that selectively targets the epidermal growth factor receptor tyrosine kinase. Cancer Biother. Radiopharm. 25, 479-485 (2010).

11. Santra, S., Kaittanis, C., Santiesteban, O. J. \& Perez, J. M. Cell-specific, activatable, and theranostic prodrug for dual-targeted cancer imaging and therapy. J. Am. Chem. Soc. 133, 16680-16688 (2011).

12. Hamburg, M. A. Science and regulation. FDA's approach to regulation of products of nanotechnology. Science 336, 299-300 (2012).
13. Josephson, L. \& Rudin, M. Barriers to clinical translation with diagnostic drugs. J. Nucl. Med. 54, 329-332 (2013).

14. Zhang, Y., Chan, H. F. \& Leong, K. W. Advanced materials and processing for drug delivery: The past and the future. Adv. Drug Deliv. Rev. 65, 104-120 (2013).

15. Blanco, E. et al. Molecular-targeted nanotherapies in cancer: enabling treatment specificity. Mol. Oncol. 5, 492-503 (2011).

16. Prakash, S., Malhotra, M., Shao, W., Tomaro-Duchesneau, C. \& Abbasi, S. Polymeric nanohybrids and functionalized carbon nanotubes as drug delivery carriers for cancer therapy. Adv. Drug Deliv. Rev. 63, 1340-1351 (2011).

17. Schroeder, A. et al. Treating metastatic cancer with nanotechnology. Nat. Rev. Cancer. 12, 39-50 (2012).

18. O'Brien, M. E. et al. Reduced cardiotoxicity and comparable efficacy in a phase III trial of pegylated liposomal doxorubicin HCl (CAELYX/Doxil) versus conventional doxorubicin for first-line treatment of metastatic breast cancer. Ann. Oncol. 15, 440-449 (2004).

19. Ringden, O. et al. Efficacy of amphotericin B encapsulated in liposomes (AmBisome) in the treatment of invasive fungal infections in immunocompromised patients. J. Antimicrob. Chemother. 28(Suppl B): 73-82 (1991).

20. McCarthy, J. R., Perez, J. M., Bruckner, C. \& Weissleder, R. Polymeric nanoparticle preparation that eradicates tumours. Nano Lett. 5, 2552-2556 (2005).

21. Santra, S., Kaittanis, C. \& Perez, J. M. Cytochrome C encapsulating theranostic nanoparticles: a novel bifunctional system for targeted delivery of therapeutic membrane-impermeable proteins to tumours and imaging of cancer therapy. Mol. Pharm. 7, 1209-1222 (2010).

22. Santra, S., Kaittanis, C. \& Perez, J. M. Aliphatic hyperbranched polyester: a new building block in the construction of multifunctional nanoparticles and nanocomposites. Langmuir 26, 5364-5373 (2010).

23. Hrkach, J. et al. Preclinical development and clinical translation of a PSMA-Targeted docetaxel nanoparticle with a differentiated pharmacological profile. Sci. Transl. Med. 4, 128 ra139 (2012).

24. Grimm, J., Perez, J. M., Josephson, L. \& Weissleder, R. Novel nanosensors for rapid analysis of telomerase activity. Cancer Res. 64, 639-643 (2004).

25. Kaittanis, C., Boukhriss, H., Santra, S., Naser, S. A. \& Perez, J. M. Rapid and sensitive detection of an intracellular pathogen in human peripheral leukocytes with hybridizing magnetic relaxation nanosensors. PLoS One 7, e35326 (2012).

26. Kaittanis, C., Naser, S. A. \& Perez, J. M. One-step, nanoparticle-mediated bacterial detection with magnetic relaxation. Nano Lett. 7, 380-383 (2007).

27. Kaittanis, C., Nath, S. \& Perez, J. M. Rapid nanoparticle-mediated monitoring of bacterial metabolic activity and assessment of antimicrobial susceptibility in blood with magnetic relaxation. PLoS One 3, e3253 (2008).

28. Kaittanis, C., Santra, S. \& Perez, J. M. Emerging nanotechnology-based strategies for the identification of microbial pathogenesis. Adv. Drug Deliv. Rev. 62, 408-423 (2010).

29. Perez, J. M., Grimm, J., Josephson, L. \& Weissleder, R. Integrated nanosensors to determine levels and functional activity of human telomerase. Neoplasia $\mathbf{1 0}$ 1066-1072 (2008).

30. Perez, J. M., Josephson, L., O'Loughlin, T., Hogemann, D. \& Weissleder, R. Magnetic relaxation switches capable of sensing molecular interactions. Nat. Biotechnol. 20, 816-820 (2002).

31. Vander Heiden, M. G., Cantley, L. C. \& Thompson, C. B. Understanding the Warburg effect: the metabolic requirements of cell proliferation. Science 324, 1029-1033 (2009).

32. Carver, B. S. et al. Reciprocal feedback regulation of PI3K and androgen receptor signaling in PTEN-deficient prostate cancer. Cancer Cell 19, 575-586 (2011).

33. Haun, J. B. et al. Micro-NMR for rapid molecular analysis of human tumour samples. Sci. Transl. Med. 3, 71ra16 (2011).

34. Issadore, D. et al. Miniature magnetic resonance system for point-of-care diagnostics. Lab. Chip. 11, 2282-2287 (2011).

35. Caravan, P. in Molecular and Cellular MR Imaging (eds Modo, M. M. J. \& Bulte, J. W. M.) 13-36 (CRC Press, 2007).

36. Ahrens, E. T. \& Bulte, J. W. Tracking immune cells in vivo using magnetic resonance imaging. Nat. Rev. Immunol. 13, 755-763 (2013).

37. Koh, I., Hong, R., Weissleder, R. \& Josephson, L. Nanoparticle-target interactions parallel antibody-protein interactions. Anal. Chem. 81, 3618-3622 (2009).

38. Kaittanis, C., Santra, S., Santiesteban, O. J., Henderson, T. J. \& Perez, J. M. The assembly state between magnetic nanosensors and their targets orchestrates their magnetic relaxation response. J. Am. Chem. Soc. 133, 3668-3676 (2011).

39. Taktak, S., Sosnovik, D., Cima, M. J., Weissleder, R. \& Josephson, L. Multiparameter magnetic relaxation switch assays. Anal. Chem. 79, 8863-8869 (2007).

40. Aslan, K., Lakowicz, J. R. \& Geddes, C. D. Nanogold-plasmon-resonance-based glucose sensing. Anal. Biochem. 330, 145-155 (2004). 
41. Yoshizumi, A., Kanayama, N., Maehara, Y., Ide, M. \& Kitano, H. Self-assembled monolayer of sugar-carrying polymer chain: Sugar balls from 2-methacryloyloxyethyl D-glucopyranoside. Langmuir. 15, 482-488 (1999).

42. Santra, S., Kaittanis, C., Grimm, J. \& Perez, J. M. Drug/dye-loaded, multifunctional iron oxide nanoparticles for combined targeted cancer therapy and dual optical/magnetic resonance imaging. Small 5, 1862-1868 (2009).

43. Nath, S., Kaittanis, C., Ramachandran, V., Dalal, N. \& Perez, J. M. Synthesis, magnetic characterization and sensing applications of novel dextran-coated iron oxide nanorods. Chem. Mater. 21, 1761-1767 (2009).

\section{Acknowledgements}

We thank Professor Lewis Cantley (Weill Cornell Medical College, Cornell University), Professor Charles Sawyers (MSKCC) and Dr Horst Kessler (Technische Universität München) for providing chemotherapeutics; Dr Jason S. Lewis for providing animals; Dr Naga Vara Kishore Pillarsetty, Dr Carl Le, Dr Simone Alidori, Dr Stefan Harmsen, Kuntalkumar Sevak, Dr Priyanka Shukla and Florian Rechenmacher for expert technical assistance. This study was supported by the Department of Defense Prostate Cancer Research Program of the Congressionally Directed Medical Research Programs under Award No. W81XWH-12-1-0509 to J.G. (opinions, interpretations, conclusions and recommendations are those of the author and are not necessarily endorsed by the funding agency), Louis V. Gerstner Young Investigator Award (to J.G.), Starr Cancer Consortium (I4-A427 to J.G.), the MSKCC Experimental Therapeutics Center (to J.G.), Mr William H. and Mrs Alice Goodwin (to J.G.), the Alex's Lemonade Stand Foundation (to C.K.) and the NIH (P30 CA008748-44 S5). T.M.S. is funded by a National Science Foundation Integrative Graduate Education and Research Traineeship (MAD, IGERT 0965983).

\section{Author contributions}

C.K. and J.G. contributed the original idea. C.K., J.M.P., L.J. and J.G. designed the experiments. J.G. supervised the project. C.K., T.M.S., A.O. and S.S. performed the experiments. Y.L. and G.C. provided materials, and all authors contributed to discussions regarding the research. C.K. and J.G. wrote the manuscript.

\section{Additional information}

Supplementary Information accompanies this paper at http://www.nature.com/ naturecommunications

Competing financial interests: The authors declare no competing financial interests.

Reprints and permission information is available online at http://www.npg.nature.com/ reprintsandpermissions/

How to cite this article: Kaittanis, C. et al. Environment-responsive nanophores for therapy and treatment monitoring via molecular MRI quenching. Nat. Commun. 5:3384 doi: $10.1038 /$ ncomms4384 (2014). 


\title{
Author Correction: Environment-responsive nanophores for therapy and treatment monitoring via molecular MRI quenching
}

\author{
Charalambos Kaittanis ${ }^{1}$, Travis M. Shaffer ${ }^{1,2}$, Anuja Ogirala ${ }^{1}$, Santimukul Santra ${ }^{3}$, J. Manuel Perez ${ }^{4}$, \\ Gabriela Chiosis ${ }^{1}$, Yueming $\mathrm{Li}^{1}$, Lee Josephson ${ }^{5}$ \& Jan Grimm ${ }^{1}$
}

Correction to: Nature Communications https://doi.org/10.1038/ncomms4384, published online 04 March 2014

This Article contains an error in Fig. 6. In panel b, the left-hand image is mistakenly described as showing fluorescence before treatment, while it in fact shows the same white light image as the right-hand panel without fluorescent overlay to better visualize the tumour location. A correct version of Fig. $6 \mathrm{~b}$ is presented below as Figure 1. The error has not been corrected in the original version of the Article.

Published online: 18 April 2019

\begin{abstract}
(c) Open Access This article is licensed under a Creative Commons Attribution 4.0 International License, which permits use, sharing, adaptation, distribution and reproduction in any medium or format, as long as you give appropriate credit to the original author(s) and the source, provide a link to the Creative Commons license, and indicate if changes were made. The images or other third party material in this article are included in the article's Creative Commons license, unless indicated otherwise in a credit line to the material. If material is not included in the article's Creative Commons license and your intended use is not permitted by statutory regulation or exceeds the permitted use, you will need to obtain permission directly from the copyright holder. To view a copy of this license, visit http://creativecommons.org/licenses/by/4.0/.
\end{abstract}

(c) The Author(s) 2019

\footnotetext{
${ }^{1}$ Molecular Pharmacology and Chemistry Program, Memorial Sloan Kettering Cancer Center, 1275 York Avenue, New York, New York 10065, USA. ${ }^{2}$ Department of Chemistry, Hunter College of the City University of New York, Graduate Center, New York, New York 10065, USA. ${ }^{3}$ Department of Chemistry, Pittsburg State University, 1701 S Broadway Street, Pittsburg, Kansas 66762, USA. ${ }^{4}$ NanoScience Technology Center, University of Central Florida, 12424 Research Parkway, Suite 400, Orlando, Florida 32826, USA. ${ }^{5}$ Center for Advanced Medical Imaging Sciences, Massachusetts General Hospital, Building 149, 13th Street,

Charlestown, Massachusetts 02129, USA. Correspondence and requests for materials should be addressed to J.G. (email: grimmj@mskcc.org)
} 


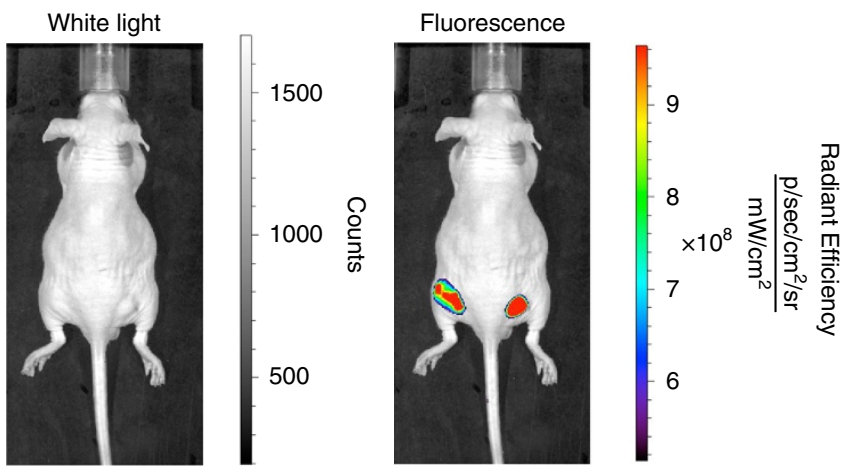

Fig. 1 